\title{
A clinical perspective on plasma cell leukemia; current status and future directions
}

\author{
Sherilyn A. Tuazon (1) 1,2, Leona A. Holmberg ${ }^{1,2}$, Omar Nadeem ${ }^{3,4}$ and Paul G. Richardson (1) ${ }^{3,4}$
}

\begin{abstract}
Primary plasma cell leukemia ( $\mathrm{pPCL}$ ) is an aggressive plasma cell disorder with a guarded prognosis. The diagnosis is confirmed when peripheral blood plasma cells (PCs) exceed 20\% of white blood cells or 2000/ $\mu \mathrm{L}$. Emerging data demonstrates that patients with lower levels of circulating (PCs) have the same adverse prognosis, challenging the clinical disease definition, but supporting the adverse impact of circulating PCs. The cornerstone of treatment consists of combination therapy incorporating a proteasome inhibitor, an immunomodulatory agent, steroids, and/or anthracyclines and alkylators as part of more-intensive chemotherapy, followed by consolidative autologous hematopoietic cell transplantation in eligible patients and then maintenance therapy. Monoclonal antibodies are also currently being evaluated in this setting with a strong rationale for their use based on their activity in multiple myeloma (MM). Due to limited therapeutic studies specifically evaluating $\mathrm{PPCL}$, patients with $\mathrm{PPCL}$ should be considered for clinical trials. In contrast to MM, the outcomes of patients with PPCL have only modestly improved with novel therapies, and secondary PCL arising from MM in particular is associated with a dismal outlook. Newer drug combinations, immunotherapy, and cellular therapy are under investigation, and these approaches hopefully will demonstrate efficacy to improve the prognosis of $\mathrm{PPCL}$.
\end{abstract}

\section{Introduction}

Plasma cell leukemia (PCL) is the rarest yet most aggressive plasma cell disorder. PCL was originally defined by an absolute plasma cell (PC) count greater than $2 \times 10^{9} / \mathrm{L}$ in the peripheral blood (PB) and more than $20 \%$ circulating $\mathrm{PCs}^{1}$. At present, only one of the original two requirements is used to define PCL according to the criteria by the World Health Organization and the International Myeloma Working Group (IMWG) ${ }^{2,3}$. The definition of PCL is currently an area of controversy as recent studies suggest that the presence of $\geq 5 \%$ circulating abnormal PCs but not meeting the 20\% cutoff have a similar adverse prognostic impact and poor survival as patients with $>20 \%$ abnormal $\mathrm{PCs}^{4,5}$. Therefore, patients

Correspondence: Sherilyn A. Tuazon (stuazon@fredhutch.org)

${ }^{1}$ Clinical Research Division, Fred Hutchinson Cancer Research Center, Seattle, WA, USA

${ }^{2}$ Department of Medicine, Medical Oncology, University of Washington, Seattle, WA, USA

Full list of author information is available at the end of the article with PCs comprising $\geq 5 \%$ of the PB white cell differential count may be considered as having PCL and can be treated as such. Another limitation of the current diagnostic definition of PCL is that it does not take into consideration plasma cell clonality, which can be characterized using multiparametric flow cytometry ${ }^{6}$. Establishing the presence of a malignant clonal population is important as reactive plasmacytosis can occur due to a variety of infections, neoplastic or inflammatory conditions $^{7-10}$.

PCL is referred to as primary when the leukemic phase presents at diagnosis, or secondary when leukemic progression occurs in the context of preexisting multiple myeloma (MM). Historically, $60-70 \%$ of cases have been reported to be primary PCL (pPCL), whereas $\sim 40 \%$ are secondary ${ }^{11}$. In recent years, there has been an increase in the incidence of secondary $\mathrm{PCL}^{12}$, likely related to more effective therapies contributing to both improved survival and clonal selection over time. In this review, we will

\section{(c) The Author(s) 2021}

(c) (i) Open Access This article is licensed under a Creative Commons Attribution 4.0 International License, which permits use, sharing, adaptation, distribution and reproduction cc) in any medium or format, as long as you give appropriate credit to the original author(s) and the source, provide a link to the Creative Commons license, and indicate if changes were made. The images or other third party material in this article are included in the article's Creative Commons license, unless indicated otherwise in a credit line to the material. If material is not included in the article's Creative Commons license and your intended use is not permitted by statutory regulation or exceeds the permitted use, you will need to obtain permission directly from the copyright holder. To view a copy of this license, visit http://creativecommons.org/licenses/by/4.0/. 
focus on pPCL, and also comment on secondary PCL, when applicable.

\section{Clinical and laboratory manifestations of PCL}

In comparison to MM, pPCL has distinct biological and clinical features. Because pPCL is rare, data has been primarily obtained from retrospective studies with small numbers of patients. The median age at diagnosis of pPCL is 61 years, approximately 10 years younger than the average age of diagnosis of a typical MM patient ${ }^{13} \cdot \mathrm{pPCL}$ is characterized by significant anemia, thrombocytopenia, renal insufficiency, hypercalcemia, and increased tumor burden (reflected by elevated lactate dehydrogenase $(\mathrm{LDH})$ and $\beta 2$-microglobulin, as well as marked bone marrow (BM) plasma cell infiltration ${ }^{2,14}$. Light chain and nonsecretory subtypes are more commonly observed in $\mathrm{pPCL}^{12}$. Extramedullary involvement is similarly more common in PPCL, and may be related to tumor cells having reduced expression of adhesion molecules (CD56, LFA-1, LFA-3, VLA-5), which impair retention of PCs within the $\mathrm{BM}^{15-18}$. Conversely, lytic bone lesions are less common in PPCL than in $\mathrm{MM}^{12}$.

The immunophenotype of MM and pPCL express both CD38 and CD138; however, pPCL cells have higher expression of CD20, CD27, CD28, and CD45 and lower expression of CD9, CD56, CD117, and HLA-DR compared to $M M^{19-21}$. The karyotype of pPCL frequently demonstrates hypodiploidy, associated with poor prognosis $^{22,23}$. Chromosome 1 aberrations (deletion $1 \mathrm{p}$ and $1 \mathrm{q}$ amplification), now considered adverse cytogenetic abnormalities by the IMWG in $\mathrm{MM}^{24}$, are also more frequent in $\mathrm{pPCL}^{25,26}$. Importantly, deletion $17 \mathrm{p}$, a notoriously high-risk marker, is observed in up to $50 \%$ of $\mathrm{pPCL}^{12}$. The most common abnormality in pPCL is $t$ $(11 ; 14)$ with a frequency ranging from $25 \%$ to $70 \%$, which in comparison, is described in only $15-20 \%$ of patients with $\mathrm{MM}^{2,10,12,27}$.

\section{Prognosis}

As in MM, the presence of adverse cytogenetic abnormalities is a major determinant of worse prognosis. Age $\geq 60$ years, platelet count $\leq 100 \times 10^{9 /} \mathrm{L}$, and PB PC count $\geq 20 \times 10^{9} / \mathrm{L}$ have also been reported as predictors of inferior survival ${ }^{28}$. Historically, patients with pPCL have a median overall survival (OS) ranging from 4 to 11 months $^{12,29}$. Widespread use of novel agents and autologous stem cell transplantation (ASCT) have modestly improved survival. This is illustrated by SEER data reporting median OS of 5, 6, 4, and 12 months in 445 patients diagnosed with pPCL during 1973-1995, 1996-2000, 2001-2005, and 2006-2009, respectively ${ }^{30}$. In patients undergoing ASCT, survival may be 2-3 years, which is still inferior and only a fraction of what can now be seen in other MM populations ${ }^{31-33}$.

\section{Diagnostic evaluation}

The initial diagnostic workup of pPCL is similar to those performed in $\mathrm{MM}$. This includes a $\mathrm{CBC} /$ differential, creatinine, calcium, LDH, $\beta 2$-microglobulin, albumin, serum protein electrophoresis/immunofixation, serumfree light chains, $24 \mathrm{~h}$ urine collection for total protein, electrophoresis/immunofixation, and BM aspiration and biopsy $^{34}$. In addition, a PET-CT should be obtained due to the high incidence of extraosseous plasmacytomas. BM specimens should be sent for morphology, flow cytometry, and cytogenetics by FISH $^{35}$. Laboratory tests to assess tumor lysis syndrome (TLS) should also be obtained $^{35}$. A lumbar puncture for cerebrospinal fluid cytology and flow cytometry can be performed, if there is any suspicion for central nervous system or leptomeningeal involvement based on clinical presentation (e.g., headache, cranial nerve palsies, marked PB leukocytosis, and large plasmacytomas appearing to encroach the brain or spinal cord on radiographs).

\section{Treatment}

Due to the aggressive nature of pPCL, immediate disease control is warranted to prevent disease-related complications and early mortality. Patients with detectable circulating PCs by conventional blood count, despite not meeting the arbitrary cutoff of $20 \%$, should be considered for treatment similar to pPCL. Because there have been no randomized prospective trials that specifically evaluate the treatment of $\mathrm{pPCL}$, therapeutic recommendations are largely based on small prospective and retrospective studies, and extrapolated data from MM trials. Importantly, enrollment to clinical trials is strongly encouraged, especially those incorporating monoclonal antibodies (mAbs) and also other targeted agents (e.g., venetoclax). Figure 1 summarizes a proposed treatment algorithm for pPCL.

\section{Induction}

Regimens that comprise the proteasome inhibitor (PI), bortezomib induce high overall response rates (ORR) of up to $79 \%$ in $\mathrm{pPCL}$ as demonstrated in retrospective studies $^{28,36,37}$. A phase II study by the IFM demonstrated an ORR of $69 \%(n=27)$ after induction with alternating cycles of bortezomib, dexamethasone + cyclophosphamide, or doxorubicin among newly diagnosed patients with $\mathrm{PPCL}^{33}$. As in MM, bortezomib may partially abrogate the adverse prognosis of high-risk cytogenetic abnormalities ${ }^{38,39}$, commonly seen in pPCL.

Among the immunomodulatory drugs (IMiDs), lenalidomide is the agent with most data demonstrating efficacy in PPCL. A phase II multicenter study showed an ORR of $60 \%$ among patients treated with lenalidomide + dexamethasone with/without high-dose melphalan supported by $\mathrm{ASCT}^{40}$. Importantly, next-generation PIs (carfilzomib, 


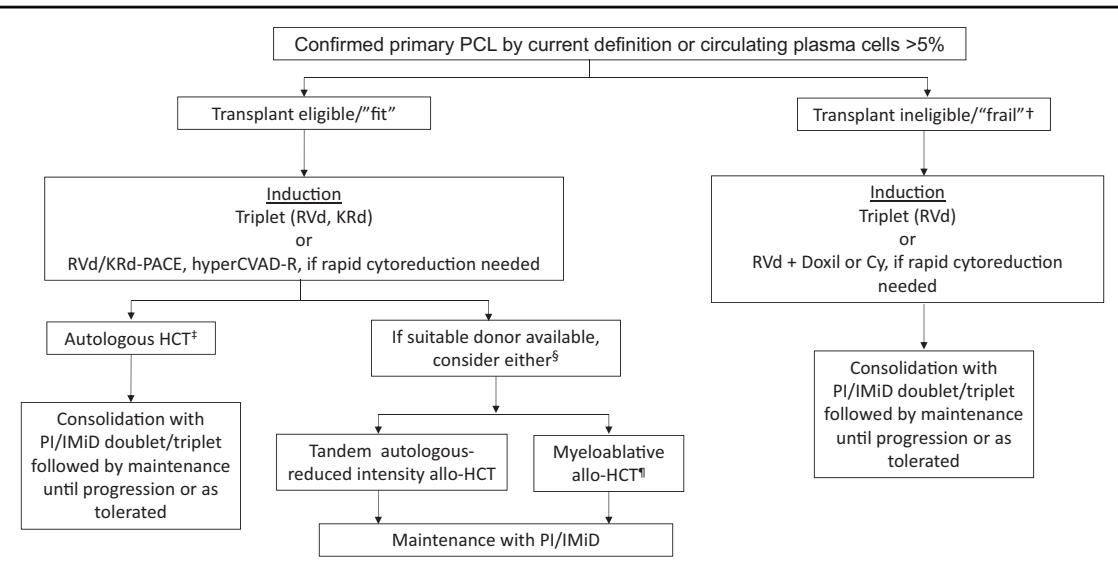

Fig. 1 Proposed treatment algorithim for plasma cell leukemia.

ixazomib), IMiDs (pomalidomide), and CD38-directed $\mathrm{mAb}$ (daratumumab) have exhibited high efficacy in MM, even among those with adverse cytogenetics. It may be reasonable to speculate that these drugs would have efficacy in PPCL. However, the data on the efficacy of these drugs is exceedingly limited as patients with pPCL are often excluded from prospective trials of MM. Recently, the European Myeloma Network presented an abstract reporting an interim analysis of a phase II trial (EMN12/ HOVON 129) using carfilzomib, lenalidomide, and dexamethasone (KRd) as induction, consolidation, and maintenance in patients with $\mathrm{pPCL}^{35}$. In patients $\leq 65$ years, 4 cycles of KRd is followed by tandem ASCT (or tandem auto-allo SCT if there is a suitable donor), KRd consolidation, followed by KR maintenance until progression. Patients $>65$ years old received 8 cycles of KRd followed by KR. Among 14 patients who received 4 cycles of KRd induction, very good partial response or greater response was observed in $80 \%$ with $33 \%$ achieving at least a complete response. The study suggests that the induction phase could be optimized by incorporating newer agents used in MM to further improve the quality of responses.

For the above reasons, combination therapies incorporating a PI and IMiD are considered frontline in the treatment of pPCL, building on the success of the lenalidomide, bortezomib, and dexamethasone platform $(\mathrm{RVd})^{41,42}$. In patients who are transplant-eligible, induction therapy should be given until best response is achieved. In those who are transplant-ineligible, between 8 and 12 cycles of induction should be administered prior to maintenance therapy.

Although limited evidence exists, intensive chemotherapy regimens, such as VTd/RVd-PACE (bortezomib, thalidomide or lenalidomide, dexamethasone, cisplatin, doxorubicin, cyclophosphamide, etoposide) or hyperCVAD-RV (cyclophosphamide, vincristine, doxorubicin, dexamethasone, lenalidomide, bortezomib) can be used for induction if rapid cytoreduction is required ${ }^{2,43-45}$. Less-intensive regimens such as $\mathrm{RVd}+$ liposomal doxorubicin or $\mathrm{RVd}+$ cyclophosphamide $^{46,47}$ are also reasonable options to be considered, particularly in frail patients ${ }^{48}$.

Novel MM therapies that are under investigation in pPCL include combination of pomalidomide with ixazomib (NCT02547662), and daratumumab combined with bortezomib, dexamethasone, pegylated liposomal doxorubicin, and lenalidomide (NCT03591744). The remarkable efficacy of the RVd + daratumumab quadruplet as induction therapy achieving deep responses in MM in the GRIFFIN trial strongly advocates for this approach in pPCL, as does the preliminary data with $\mathrm{KRd}+$ daratumumab $^{49,50}$. Finally, encouraging data using venetoclax targeting $\mathrm{t}(11 ; 14) \mathrm{MM}$ provides a clear rationale for incorporating this approach in PPCL, with clinical trials in development for combinations in the newly diagnosed setting $^{51,52}$.

\section{Hematopoietic cell transplantation}

Phase II trials have demonstrated that PI and IMiDs combined with ASCT may be effective in a proportion of patients with $\mathrm{pPCL}^{33,40}$. The feasibility and potential efficacy of ASCT in PPCL are also demonstrated in registry studies. The European Group for Blood and Marrow Transplantation reported outcomes of 272 patients with pPCL who underwent ASCT between 1980 and 2006. The median progression-free survival (PFS) and median OS were 14.3 months and 15.7 months, respectively. Data from the Center for International Blood and Marrow Transplant Research (CIBMTR) demonstrated a 3 year PFS and 3 year OS of $34 \%$ and $64 \%$, respectively, among 97 patients with pPCL who underwent $\mathrm{ASCT}^{32}$. Furthermore, there was a trend toward superior OS in patients who underwent a tandem compared to a single $\mathrm{ASCT}^{32}$. Recent data from the CIBMTR demonstrated 
persistently poor post-ASCT outcomes in 348 patients with pPCL despite the widespread use of novel agents. The 3-year OS was 35\% and 38\% in patients after ASCT and allo-SCT, respectively ${ }^{53}$. Despite the potential benefit of ASCT, it is clear that intensification of therapy with novel combination regimens in conjunction with ASCT, if appropriate, is still needed to improve pPCL outcomes, as well as innovative approaches to maintenance therapy. One way to intensify treatment is with tandem ASCT, which although largely unproven in pPCL may improve outcomes as demonstrated by better PFS in patients with high-risk MM in the EMN02/HO95 ${ }^{54}$ and updated results of the STaMINA trial ${ }^{55}$.

Despite the possibility of cure, there have been variable reports on the efficacy of allo-SCT in pPCL. The CIBMTR reported the outcomes of 50 patients with pPCL who underwent an allo-SCT, the majority of whom (68\%) received a myeloablative conditioning regimen ${ }^{32}$. The cumulative incidence of relapse at 3 years was significantly lower with allo-SCT (38\% vs. 61\%) compared with ASCT but the 3-year OS was inferior with allo-SCT $(39 \%$ vs. $64 \%)^{32}$. The lack of OS benefit with allo-SCT could partly be due to a higher treatment-related mortality (TRM) $(41 \% \text { allo-SCT vs. } 5 \% \text { in the ASCT })^{32}$. Survival of patients treated with allo-SCT plateaued at $20 \%$ in 5 years suggesting that a proportion of patients with pPCL can achieve long-term remissions with this approach. In the IFM trial, 16 responding patients $\leq 65$ years with an HLAmatched donor received a tandem ASCT and reducedintensity conditioning allo-SCT. Six patients who did not meet criteria for allo-SCT proceeded to a second ASCT followed by consolidation/maintenance with RVd for 1 year. After a median follow-up of 28.7 months, the median PFS and OS were 15.1 and 36.3 months, respectively ${ }^{33}$.

Because allo-SCT is associated with dismal OS when applied after relapse, it should be offered early, if considered at all $^{56}$. As demonstrated in patients with MM, allo-SCT is also fraught by high rates of TRM, particularly with myeloablative conditioning regimens ${ }^{57,58}$. Lessintensive conditioning regimens for allo-SCT that rely on graft versus myeloma (GvM) effects for disease eradication, significantly reduce TRM but at the expense of higher relapse rates ${ }^{59}$. Myeloablative ASCT followed by reduced-intensity allo-SCT (tandem auto/allo-SCT) allows for optimal cytoreduction followed by the immunologic GvM, and in the context of protocol-directed therapy, warrants consideration as part of prospective studies.

\section{Maintenance}

pPCL is characterized by short remissions and early relapse; therefore, early institution of maintenance therapy and/or consolidation post-ASCT ( day $60-80)$ is recommended to prevent disease progression. Singleagent maintenance appears insufficient to maintain remissions in PPCL as demonstrated by $80 \%$ relapse rate occurring at 2-12 months of lenalidomide maintenance ${ }^{40}$. Growing evidence indicates that consolidation with a doublet or triplet may improve response rates and PFS in $\mathrm{MM}^{60}$, which may support similar strategies in PPCL such as lenalidomide combined with bortezomib, with or without a mAb.

Maintenance therapy after allo-SCT is controversial but reasonable to administer while awaiting full GvM effect. Lenalidomide may potentiate acute graft-versus hostdisease (GvHD) when instituted early after allo-SCT, but may be effective at a lower dose ${ }^{61,62}$. On the other hand, bortezomib is safe and feasible when administered postallo-SCT ${ }^{63}$, and may decrease the risk of GvHD, partly by impairing the activation of $\mathrm{T}$-cells and antigen-presenting cells ${ }^{64,65}$.

\section{Supportive care}

During treatment initiation, patients with pPCL are at risk for TLS due to a high tumor burden and rapid cell turnover. TLS precautions should be instituted with adequate hydration and prophylaxis with allopurinol or rasburicase (if high-risk). For patients on PIs, varicella zoster prophylaxis should be administered concurrently. Patients should receive venous thromboembolism prophylaxis while on IMiDs. Although osteolytic lesions are less frequent in PPCL relative to MM, all patients with pPCL should be started on anti-resorptive bone targeting agents to reduce the risk of skeletal-related events. Hydration to preserve renal function is also critical, as is more broadly, caution regarding increased risk of infections with particular attention to growth factor use, IVIG administration, and antibiotics as clinically indicated.

\section{Emerging therapies for PCL}

As mentioned, $t(11 ; 14)$ is frequently observed in pPCL and is a marker of BCL-2 overexpression; thus, venetoclax (BCL-2 inhibitor) may be useful. In phase I trials of patients with relapsed/refractory $M M$, venetoclax has demonstrated high responses as a single agent (ORR $40 \%)^{66}$ and in combination with bortezomib and dexamethasone (ORR $78 \%)^{67}$ in patients harboring $t(11 ; 14)$. In patients with relapsed pPCL, case reports have demonstrated efficacy of venetoclax as a single agent ${ }^{68}$ or in combination with daratumumab, dexamethasone with $^{69}$ or without bortezomib ${ }^{70}$.

Chimeric antigen receptor (CAR) T-cell therapy ${ }^{71}$ and other immunotherapies (e.g., bispecific $\mathrm{T}$-cell engagers ${ }^{72}$, antibody-drug conjugates ${ }^{73}$ ) that target B-cell maturation antigen (BCMA) have gained momentum in the recent years due to striking initial responses even among highrisk and heavily pretreated individuals with MM. 


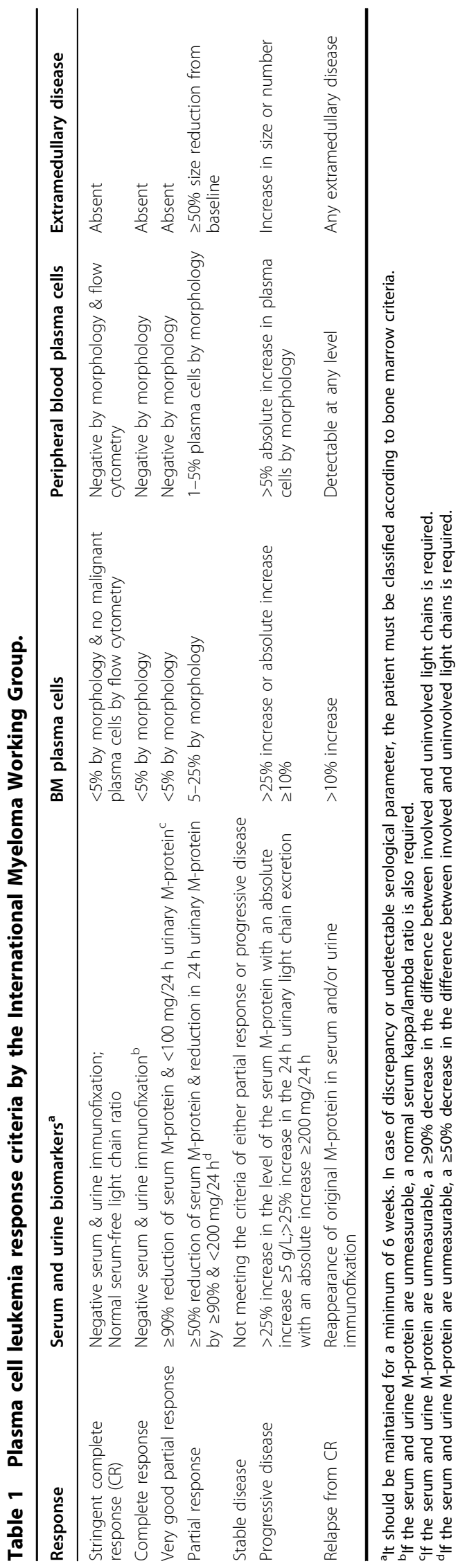

However, the ability to generate durable functional antimyeloma T-cell responses is still limited. In a phase I study of bb2121 (BCMA-targeted CAR T-cell), the ORR was $85 \%$ among 33 treated patients, including 15 patients with complete responses with median PFS of 11.8 months $^{71}$. The role of CAR T-cells and other immunotherapeutic strategies in the treatment of pPCL remains to be defined.

Other emerging therapies for PPCL include a phase II trial of NK cells in combination with elotuzumab, lenalidomide, and high-dose melphalan before ASCT (NCT01729091) and a phase II trial of panobinostat, gemcitabine, busulfan, and melphalan before ASCT (NCT02506959). In this context, the use of panobinostat in combination with other novel agents including mAbs has a strong rationale; as does the incorporation of selinexor into multiagent regimens, especially given the promising activity of this next-generation novel drug in targeting del $(17 p)^{74,75}$.

\section{Assessing treatment response}

In contrast to $\mathrm{MM}$, there are no specific treatment response criteria for PCL. The response criteria used in MM appears inadequate to assess response in PCL due to the leukemic nature of the disease, increased incidence of light chain and nonsecretory forms, and extramedullary disease. The IMWG proposed the inclusion of PB PC criterion and assessment of extramedullary disease (by PET-CT) to the standard biomarker and BM criteria (Table 1$)^{2}$. In case of undetectable BM and PB PCs by morphology, flow cytometry should be used to measure residual disease.

\section{Treatment of relapsed or refractory $\mathrm{PCL}$, including secondary PCL}

Limited data exists to guide treatment for relapsed/ refractory pPCL. Clinical trial participation should be encouraged if an appropriate study is available; although pPCL is typically an exclusive criteria to most studies in the advanced disease setting. A critical option is to utilize a combination of active drugs in MM, particularly ones that the patient has not previously received nor is refractory to. Patients who experience a deep and prolonged response to a prior therapy, re-treatment with the same regimen can be considered, particularly if the relapse occurred off therapy ${ }^{76}$.

For patients in whom RVd-based therapy has been utilized initially, carfilzomib-based treatment can be deployed at relapse; similarly, if MM disease progression manifesting as secondary PCL occurs, the use of carfilzomib-based therapy should also be used in the same context. This sequencing of therapeutic strategies has been recently validated by the events of the ENDURANCE trial comparing RVd to KRd in the standard newly 
diagnosed MM patient where equivalent outcomes and less cardiovascular, renal, and pulmonary toxicity was seen with $\mathrm{RVd}^{77}$.

Salvage strategies using combinations of novel agents incorporating pomalidomide, panobinostat, elotuzumab, daratumumab, isatuximab, and if appropriate ixazomib, selinexor, and belantamab mafodotin can all be considered $^{78-85}$. Combination regimens incorporating chemotherapy are also a potentially mainstay in this frequently difficult-to-treat population, and the use of high-dose steroids as well as nonmyelotoxic agents such as thalidomide as part of these approaches can have merit $^{86,87}$.

It is hoped that additional approaches can be engendered from the next wave of novel agents, and especially those targeting "stemness" and extramedullary disease, such as melflufen, with current studies informing realworld practice ${ }^{88-90}$.

\section{Conclusion}

The use of multidrug combinations (including a PI, an $\mathrm{IMiD}$, and now a mAb) for induction appears to be a rational approach to consider in pPCL. While rates of post-ASCT relapse remain high, ASCT should be incorporated in eligible patients when appropriate, either as tandem ASCT or tandem ASCT/allo-SCT, comparable to the paradigm used more broadly in relapsed/refractory $M M$, given the need for sustained response. Similarly, because of the high TRM, myeloablative allo-SCT should only be performed in the context of a trial. Consolidation and maintenance until disease progression should be administered to both transplant-eligible and ineligible patients. Although enrollment to clinical trials is strongly advised, this is not always feasible partly due to the relative paucity of specific studies on pPCL, highlighting the critical need for prospective trials for this especially challenging high-risk subgroup. The role of immunotherapies (CAR T-cells, mAbs, bispecific T-cell engagers, antibody-drug conjugates) and small molecule inhibitors, such as venetoclax, in the treatment of PPCL is much needed and eagerly awaited.

\section{Acknowledgements}

The authors gratefully acknowledge the editorial assistance of Ashley Ford and Jack Sparacino, supported in part by the R.J. Corman Multiple Myeloma Research Foundation. Funding source: S.A.T. is supported by grants from the Conquer Cancer Foundation and the NIH/NCI P30 CA015704.

\section{Author details}

${ }^{1}$ Clinical Research Division, Fred Hutchinson Cancer Research Center, Seattle, WA, USA. ${ }^{2}$ Department of Medicine, Medical Oncology, University of Washington, Seattle, WA, USA. ${ }^{3}$ Dana-Farber Cancer Institute, Boston, MA, USA. ${ }^{4}$ Harvard Medical School, Boston, MA, USA

\section{Conflict of interest}

S.A.T. does not have any conflict of interest. L.A.H reports research funding from Seattle Genetics, Merck, Millennium Takeda Pharmaceuticals, Celgene/
BMS, Sanofi-Aventis and Janssen, and royalties from UpToDate. P.G.R. is an advisory committee member for Karyopharm, Oncopeptides, Celgene/BMS, Takeda, Janssen Sanofi, SecuraBio and GSK, and receives research grant support from Oncopeptides, Celgene/BMS, and Takeda. O.N. is an advisory committee member for Takeda, Celgene/BMS, Sanofi, GSK, and has received consulting fees from Janssen.

\section{Publisher's note}

Springer Nature remains neutral with regard to jurisdictional claims in published maps and institutional affiliations.

Received: 13 October 2020 Revised: 15 December 2020 Accepted: 14 January 2021

Published online: 04 February 2021

\section{References}

1. Kyle, R. A., Maldonado, J. E. \& Bayrd, E. D. Plasma cell leukemia. Report on 17 cases.Arch. Intern. Med. 133, 813-818 (1974).

2. Fernandez de Larrea, $C$ et al. Plasma cell leukemia: consensus statement on diagnostic requirements, response criteria and treatment recommendations by the International Myeloma Working Group. Leukemia. 27, 780-791 (2013).

3. Swerdlow S. H. C. E., et al. WHO Classification of Tumours of Haematopoietic and Lymphoid Tissues. Revised 4th edn. (WHO, 2017).

4. Granell, M. et al. Prognostic impact of circulating plasma cells in patients with multiple myeloma: implications for plasma cell leukemia definition. Haematologica. 102, 1099-1104 (2017).

5. Ravi, P. et al. Revised diagnostic criteria for plasma cell leukemia: results of a Mayo Clinic study with comparison of outcomes to multiple myeloma. Blood Cancer J. 8, 116 (2018).

6. Evans, L.A. et al. Utilizing multiparametric flow cytometry in the diagnosis of patients with primary plasma cell leukemiaAm. J. Hematol. 95, 637-642 (2020).

7. Zhang, J. et al. Reactive plasmacytosis mimicking multiple myeloma associated with SFTS virus infection: a report of two cases and literature review. BMC Infect. Dis. 18, 528 (2018).

8. Shtalrid, M., Shvidel, L. \& Vorst, E. Polyclonal reactive peripheral blood plasmacytosis mimicking plasma cell leukemia in a patient with Staphylococcal sepsis. Leuk. Lymphoma. 44, 379-380 (2003).

9. Okabe, M., Hirono, K., Tamura, K., Ichida, F. \& Kanegane, H. Reactive peripheral blood plasmacytosis in Kawasaki disease. Pediatr. Int. 60, 884-885 (2018).

10. Touzeau, C. et al. Reactive plasmacytoses can mimick plasma cell leukemia: therapeutical implications. Leuk. Lymphoma. 48, 207-208 (2007).

11. Pagano, L. et al. Primary plasma cell leukemia: a retrospective multicenter study of 73 patients. Ann. Oncol. 22, 1628-1635 (2011).

12. Tiedemann, R. E. et al. Genetic aberrations and survival in plasma cell leukemia Leukemia. 22, 1044-1052 (2008).

13. Gundesen, M. T., Lund, T., Moeller, H. E. H. \& Abildgaard, N. Plasma cell leukemia: definition, presentation, and treatment. Curr. Oncol. Rep. 21, 8 (2019).

14. Drake, M. B. et al. Primary plasma cell leukemia and autologous stem cell transplantation. Haematologica. 95, 804-809 (2010).

15. Kraj, M., Kopec-Szlezak, J., Poglod, R. \& Kruk, B. Flow cytometric immunophenotypic characteristics of 36 cases of plasma cell leukemia. Leuk. Res. 35, 169-176 (2011).

16. Schmidmaier, R. et al. Inhibition of lymphocyte function associated antigen 1 by LFA878 induces apoptosis in multiple myeloma cells and is associated with downregulation of the focal adhesion kinase/phosphatidylinositol 3 kinase/Akt pathway. Int. J. Oncol. 31, 969-976 (2007).

17. Ohtake, $\mathrm{K}$. et al. Integrin VLA-5 negative primary plasma cell leukemia. Intern Med. 32, 565-568 (1993).

18. Barker, H. F., Hamilton, M. S., Ball, J., Drew, M. \& Franklin, I. M. Expression of adhesion molecules LFA-3 and N-CAM on normal and malignant human plasma cells. Br. J. Haematol. 81, 331-335 (1992).

19. Garcia-Sanz, R. et al. Primary plasma cell leukemia: clinical, immunophenotypic, DNA ploidy, and cytogenetic characteristics. Blood 93, 1032-1037 (1999).

20. Guikema, J. E., Vellenga, E., Abdulahad, W. H., Hovenga, S. \& Bos, N. A. CD27triggering on primary plasma cell leukaemia cells has anti-apoptotic effects involving mitogen activated protein kinases. Br. J. Haematol. 124, 299-308 (2004). 
21. Katodritou, E. et al. Treatment with bortezomib-based regimens improves overall response and predicts for survival in patients with primary or secondary plasma cell leukemia: analysis of the Greek myeloma study group. Am. J. Hematol. 89, 145-150 (2014).

22. Avet-Loiseau, H. et al. Cytogenetic, interphase, and multicolor fluorescence in situ hybridization analyses in primary plasma cell leukemia: a study of 40 patients at diagnosis, on behalf of the Intergroupe Francophone du Myélome and the Groupe Français de Cytogénétique Hématologique. Blood 97, 822-825 (2001).

23. Chiecchio, L. et al. Frequent upregulation of MYC in plasma cell leukemia. Genes Chromosomes Cancer 48, 624-636 (2009).

24. Sonneveld, P. et al. Treatment of multiple myeloma with high-risk cytogenetics: a consensus of the International Myeloma Working Group. Blood 127, 2955-2962 (2016).

25. Chang, $\mathrm{H}$. et al. Genetic aberrations including chromosome 1 abnormalities and clinical features of plasma cell leukemia. Leuk. Res. 33, 259-262 (2009).

26. Chang, H., Yeung, J., Xu, W., Ning, Y. \& Patterson, B. Significant increase of CKS1B amplification from monoclonal gammopathy of undetermined significance to multiple myeloma and plasma cell leukaemia as demonstrated by interphase fluorescence in situ hybridisation. Br. J. Haematol. 134, 613-615 (2006).

27. Fonseca, R. et al. Myeloma and the $t(11 ; 14)(q 13 ; q 32)$; evidence for a biologically defined unique subset of patients. Blood 99, 3735-3741 (2002).

28. Jurczyszyn, A. et al. Prognostic indicators in primary plasma cell leukaemia: a multicentre retrospective study of 117 patients. Br. J. Haematol. 180, 831-839 (2018).

29. Ramsingh, G., Mehan, P., Luo, J., Vij, R. \& Morgensztern, D. Primary plasma cell leukemia: a surveillance, epidemiology, and end results database analysis between 1973 and 2004. Cancer 115, 5734-5739 (2009).

30. Gonsalves, W. I. et al. Trends in survival of patients with primary plasma cell leukemia: a population-based analysis. Blood 124, 907-912 (2014).

31. Musto, P. Progress in the treatment of primary plasma cell leukemia. J. Clin. Oncol. 34, 2082-2084 (2016).

32. Mahindra, A. et al. Hematopoietic cell transplantation for primary plasma cell leukemia: results from the Center for International Blood and Marrow Transplant Research. Leukemia 26, 1091-1097 (2012).

33. Royer, B. et al. Bortezomib, doxorubicin, cyclophosphamide, dexamethasone induction followed by stem cell transplantation for primary plasma cell leukemia: a prospective phase II study of the Intergroupe Francophone du Myelome. J Clin Oncol. 34, 2125-2132 (2016).

34. van de Donk, N. W., Lokhorst, H. M., Anderson, K. C. \& Richardson, P. G. How I treat plasma cell leukemia. Blood 120, 2376-2389 (2012).

35. Van De Donk, N. W. C. J. et al. Treatment of primary plasma cell leukemia with carfilzomib and lenalidomide-based therapy: results of the first interim analysis of the phase 2 EMN12/HOVON129 study. Blood 134(Suppl. 1), 693- (2019).

36. D'Arena, G. et al. Frontline chemotherapy with bortezomib-containing combinations improves response rate and survival in primary plasma cell leukemia: a retrospective study from GIMEMA Multiple Myeloma Working Party. Ann. Oncol. 23, 1499-1502 (2012).

37. Katodritou, E. et al. Real-world data on prognosis and outcome of primary plasma cell leukemia in the era of novel agents: a multicenter national study by the Greek Myeloma Study Group. Blood Cancer J. 8, 31 (2018).

38. Sonneveld, P. et al. Bortezomib induction and maintenance treatment in patients with newly diagnosed multiple myeloma: results of the randomized phase III HOVON-65/ GMMG-HD4 trial. J. Clin. Oncol. 30, 2946-2955 (2012).

39. Neben, K. et al. Administration of bortezomib before and after autologous stem cell transplantation improves outcome in multiple myeloma patients with deletion 17p. Blood 119, 940-948 (2012).

40. Musto, P. et al. Lenalidomide and low-dose dexamethasone for newly diagnosed primary plasma cell leukemia. Leukemia 28, 222-225 (2014).

41. Richardson, P. G. et al. Lenalidomide, bortezomib, and dexamethasone combination therapy in patients with newly diagnosed multiple myeloma. Blood 116, 679-686 (2010).

42. Richardson, P. G. et al. A phase 2 trial of lenalidomide, bortezomib, and dexamethasone in patients with relapsed and relapsed/refractory myeloma. Blood 123, 1461-1469 (2014).

43. Lakshman, A. et al. Efficacy of VDT PACE-like regimens in treatment of relapsed/refractory multiple myeloma. Am. J. Hematol. 93, 179-186 (2018).

44. Barlogie, B. et al. Incorporating bortezomib into upfront treatment for multiple myeloma: early results of total therapy 3. Br. J. Haematol. 138, 176-185 (2007).
45. Saraceni, M. M. et al. Modified hyperCVAD versus bortezomib-hyperCAD in patients with relapsed/refractory multiple myeloma. Clin. Lymphoma Myeloma Leuk. 18, e77-e84 (2018).

46. Jakubowiak, A. J. et al. Lenalidomide, bortezomib, pegylated liposomal doxorubicin, and dexamethasone in newly diagnosed multiple myeloma: a phase 1/2 Multiple Myeloma Research Consortium trial. Blood 118, 535-543 (2011).

47. Kumar, S. et al. Randomized, multicenter, phase 2 study (EVOLUTION) of combinations of bortezomib, dexamethasone, cyclophosphamide, and lenalidomide in previously untreated multiple myeloma. Blood 119, 4375-4382 (2012).

48. Palumbo, A. et al. Geriatric assessment predicts survival and toxicities in elderly myeloma patients: an International Myeloma Working Group report. Blood 125, 2068-2074 (2015).

49. Voorhees, P. M. et al. Daratumumab, lenalidomide, bortezomib, and dexamethasone for transplant-eligible newly diagnosed multiple myeloma: the GRIFFIN trial. Blood 136, 936-945 (2020).

50. Landgren, O. et al. Carfilzomib with immunomodulatory drugs for the treatment of newly diagnosed multiple myeloma. Leukemia 33, 2127-2143 (2019).

51. Kumar, S. et al. Updated results from BELLINI, a phase III study of venetoclax or placebo in combination with bortezomib and dexamethasone in relapsed/ refractory multiple myeloma. J. Clin. Oncol. 38(Suppl. 15), 8509 (2020).

52. Costa, L. et al. Phase 2 study of venetoclax plus carfilzomib and dexamethasone in patients with relapsed/refractory multiple myeloma. J. Clin. Oncol. 36(Suppl. 15), 8004 (2018)

53. Dhakal, B. et al. Hematopoietic cell transplantation utilization and outcomes for primary plasma cell leukemia in the current era.Leukemia 34, 3338-3347 (2020).

54. Cavo, M. et al. Autologous haematopoietic stem-cell transplantation versus bortezomib-melphalan-prednisone, with or without bortezomiblenalidomide-dexamethasone consolidation therapy, and lenalidomide maintenance for newly diagnosed multiple myeloma (EMN02/HO95): a multicentre, randomised, open-label, phase 3 study. Lancet Haematol. 7, e456-e468 (2020)

55. Hari, P. et al. Long-term follow-up of BMT CTN 0702 (STaMINA) of postautologous hematopoietic cell transplantation (autoHCT) strategies in the upfront treatment of multiple myeloma (MM). J. Clin. Oncol. 38(Suppl. 15), 8506 (2020).

56. Freytes, C. O. et al. Second transplants for multiple myeloma relapsing after a previous autotransplant-reduced-intensity allogeneic vs autologous transplantation. Bone Marrow Transplant. 49, 416-421 (2014).

57. Gahrton, G. et al. Allogeneic bone marrow transplantation in multiple myeloma. European Group for Bone Marrow Transplantation. N. Engl. J. Med. 325, 1267-1273 (1991)

58. Bensinger, W. I. et al. Allogeneic marrow transplantation for multiple myeloma: an analysis of risk factors on outcome. Blood 88, 2787-2793 (1996).

59. Crawley, C. et al. Outcomes for reduced-intensity allogeneic transplantation for multiple myeloma: an analysis of prognostic factors from the Chronic Leukaemia Working Party of the EBMT. Blood 105, 4532-4539 (2005).

60. Nooka, A. K. et al. Consolidation and maintenance therapy with lenalidomide, bortezomib and dexamethasone (RVD) in high-risk myeloma patients. Leukemia 28, 690-693 (2014).

61. Kneppers, E. et al. Lenalidomide maintenance after nonmyeloablative allogeneic stem cell transplantation in multiple myeloma is not feasible: results of the HOVON 76 Trial. Blood 118, 2413-2419 (2011).

62. Alsina, $\mathrm{M}$. et al. Lenalidomide maintenance for high-risk multiple myeloma after allogeneic hematopoietic cell transplantation. Biol. Blood Marrow Transplant. 20, 1183-1189 (2014).

63. Green, D. J. et al. Tandem autologous/allogeneic hematopoietic cell transplantation with bortezomib maintenance therapy for high-risk myeloma. Blood Adv. 1, 2247-2256 (2017).

64. Nencioni, A. et al. Proteasome inhibitor bortezomib modulates TLR4-induced dendritic cell activation. Blood 108, 551-558 (2006).

65. Blanco, B. et al. Bortezomib induces selective depletion of alloreactive $T$ lymphocytes and decreases the production of Th1 cytokines. Blood 107, 3575-3583 (2006).

66. Kumar, S. et al. Efficacy of venetoclax as targeted therapy for relapsed/ refractory t(11;14) multiple myeloma. Blood 130, 2401-2409 (2017).

67. Moreau, P. et al. Promising efficacy and acceptable safety of venetoclax plus bortezomib and dexamethasone in relapsed/refractory MM. Blood 130, 2392-2400 (2017). 
68. Jelinek, T. et al. Single-agent venetoclax induces MRD-negative response in relapsed primary plasma cell leukemia with t(11;14). Am. J. Hematol. 94 E35-e7 (2019)

69. Gonsalves, W. I., Buadi, F. K. \& Kumar, S. K. Combination therapy incorporating $\mathrm{BCl}-2$ inhibition with Venetoclax for the treatment of refractory primary plasma cell leukemia with $t(11 ; 14)$. Eur. J. Haematol. 100, 215-217 (2018).

70. Nalghranyan, S., Singh, A. P. \& Schinke, C. The combination of venetoclax, daratumumab and dexamethasone for the treatment of refractory primary plasma cell leukemia. Am. J. Hematol. 95, E34-e5 (2020).

71. Raje, N. et al. Anti-BCMA CAR T-cell therapy bb2121 in relapsed or refractory multiple myeloma. N. Engl. J. Med. 380, 1726-1737 (2019).

72. Topp, M. S. et al. Anti-B-cell maturation antigen BiTE molecule AMG 420 induces responses in multiple myeloma. J. Clin. Oncol. 38, 775-783 (2020).

73. Trudel, S. et al. Targeting B-cell maturation antigen with GSK2857916 antibody-drug conjugate in relapsed or refractory multiple myeloma (BMA117159): a dose escalation and expansion phase 1 trial. Lancet Oncol. 19 1641-1653 (2018).

74. Richardson, P. G. et al. Panobinostat plus bortezomib and dexamethasone in previously treated multiple myeloma: outcomes by prior treatment. Blood 127, 713-721 (2016)

75. Chari, A. et al. Oral selinexor-dexamethasone for triple-class refractory multiple myeloma. N. Engl. J. Med. 381, 727-738 (2019).

76. Laubach, J. et al. Management of relapsed multiple myeloma: recommendations of the International Myeloma Working Group. Leukemia $\mathbf{3 0}$ 1005-1017 (2016)

77. Kumar, S. K. et al. Carfilzomib or bortezomib in combination with lenalidomide and dexamethasone for patients with newly diagnosed multiple myeloma without intention for immediate autologous stem-cell transplantation (ENDURANCE): a multicentre, open-label, phase 3, randomised, controlled trial. Lancet Oncol. 21, 1317-1330 (2020).

78. Richardson, P. G. et al. Pomalidomide, bortezomib, and dexamethasone for patients with relapsed or refractory multiple myeloma previously treated with lenalidomide (OPTIMISMM): a randomised, open-label, phase 3 trial. Lancet Oncol. 20, 781-794 (2019).
79. Popat, R. et al. Bortezomib, thalidomide, dexamethasone, and panobinostat for patients with relapsed multiple myeloma (MUK-six): a multicentre, open-label, phase 1/2 trial. Lancet Haematol. 3, e572-e580 (2016).

80. Lonial, S. et al. Elotuzumab therapy for relapsed or refractory multiple myeloma. N. Engl. J. Med. 373, 621-631 (2015).

81. Lokhorst, H. M. et al. Targeting CD38 with daratumumab monotherapy in multiple myeloma. N. Engl. J. Med. 373, 1207-1219 (2015).

82. Attal, M. et al. Isatuximab plus pomalidomide and low-dose dexamethasone versus pomalidomide and low-dose dexamethasone in patients with relapsed and refractory multiple myeloma (ICARIA-MM): a randomised, multicentre, open-label, phase 3 study. Lancet 394, 2096-2107 (2019).

83. Moreau, P. et al. Oral ixazomib, lenalidomide, and dexamethasone for multiple myeloma. N. Engl. J. Med. 374, 1621-1634 (2016).

84. Vogl, D. T. et al. Selective inhibition of nuclear export with oral selinexor for treatment of relapsed or refractory multiple myeloma. J. Clin. Oncol. 36 859-866 (2018).

85. Lonial, S. et al. Belantamab mafodotin for relapsed or refractory multiple myeloma (DREAMM-2): a two-arm, randomised, open-label, phase 2 study. Lancet Oncol. 21, 207-221 (2020).

86. Gertz, M. A., Garton, J. P., Greipp, P. R., Witzig, T. E. \& Kyle, R. A. A phase II study of high-dose methylprednisolone in refractory or relapsed multiple myeloma Leukemia 9, 2115-2118 (1995).

87. Richardson, P. et al. Thalidomide for patients with relapsed multiple myeloma after high-dose chemotherapy and stem cell transplantation: results of an open-label multicenter phase 2 study of efficacy, toxicity, and biological activity. Mayo Clin. Proc. 79, 875-882 (2004).

88. Mateos, M. et al. HORIZON (OP-106): an exploratory analysis of time-to-next treatment (TTNT) in patients (pts) with relapsed/refractory multiple myeloma (RRMM) who received melflufen plus dexamethasone (dex). J. Clin. Oncol. 38 (Suppl. 15), e20570-e20570 (2020).

89. Ocio, E. M. et al. ANCHOR (OP-104): updated efficacy and safety from a phase $1 / 2$ study of melflufen and dexamethasone plus bortezomib or daratumumab in patients with relapsed/refractory multiple myeloma (RRMM) refractory to an IMiD or a proteasome inhibitor (PI). Blood 134(Suppl. 1), 3124 (2019).

90. Richardson, P. G. et al. Interpreting clinical trial data in multiple myeloma: translating findings to the real-world setting. Blood Cancer J. 8, 109 (2018). 